ACCEPTED MANUSCRIPT

\title{
Tunable plasmon-induced transparency and slow light in terahertz chipscale semiconductor plasmonic waveguides
}

To cite this article before publication: Zhaojian Zhang et al 2020 J. Phys. D: Appl. Phys. in press https://doi.org/10.1088/1361-6463/ab8322

\section{Manuscript version: Accepted Manuscript}

Accepted Manuscript is "the version of the article accepted for publication including all changes made as a result of the peer review process, and which may also include the addition to the article by IOP Publishing of a header, an article ID, a cover sheet and/or an 'Accepted Manuscript' watermark, but excluding any other editing, typesetting or other changes made by IOP Publishing and/or its licensors"

This Accepted Manuscript is @ 2020 IOP Publishing Ltd.

During the embargo period (the 12 month period from the publication of the Version of Record of this article), the Accepted Manuscript is fully protected by copyright and cannot be reused or reposted elsewhere.

As the Version of Record of this article is going to be / has been published on a subscription basis, this Accepted Manuscript is available for reuse under a CC BY-NC-ND 3.0 licence after the 12 month embargo period.

After the embargo period, everyone is permitted to use copy and redistribute this article for non-commercial purposes only, provided that they adhere to all the terms of the licence https://creativecommons.org/licences/by-nc-nd/3.0

Although reasonable endeavours have been taken to obtain all necessary permissions from third parties to include their copyrighted content within this article, their full citation and copyright line may not be present in this Accepted Manuscript version. Before using any content from this article, please refer to the Version of Record on IOPscience once published for full citation and copyright details, as permissions will likely be required. All third party content is fully copyright protected, unless specifically stated otherwise in the figure caption in the Version of Record.

View the article online for updates and enhancements. 


\title{
Tunable plasmon-induced transparency and slow light in terahertz chipscale semiconductor plasmonic waveguides
}

\author{
Zhaojian Zhang ${ }^{\text {a }}$, Junbo Yang ${ }^{\mathrm{b},}{ }^{*}$, Xin He ${ }^{\mathrm{b}}$, Yunxin Han ${ }^{\mathrm{b}}$, Jie Huang ${ }^{\mathrm{a}}$ and Dingbo Chen ${ }^{\mathrm{a}}$ \\ ${ }^{\text {a }}$ College of Liberal Arts and Sciences, National University of Defense Technology, Changsha 410073, China \\ ${ }^{\mathrm{b}}$ Center of Material Science, National University of Defense Technology, Changsha 410073, China \\ *e-mail: yangjunbo@nudt.edu.cn
}

\begin{abstract}
We numerically propose the plasmon-induced transparency (PIT) and slow light effect based on terahertz (THz) chipscale plasmonic semiconductor-insulator-semiconductor (SIS) waveguide system. Via the coupling between two stub resonators, PIT is introduced due to the destructive interference, which can be theoretically described by the transmission-line Method (TLM). Meanwhile, the strong dispersion within the transparent window will lead to the slow light effect. Via tuning the geometric parameters, the profile of PIT as well as group delay line can be arbitrarily tailored. Moreover, active control of both PIT and slow light can be realized by changing the ambient temperature. Especially, by integrating monolayer graphene into the structure, PIT and slow light can also be electrically modulated via applying voltage. This work provides geometrical, thermal and electrical approaches to manipulate $\mathrm{THz}$ transmission and group delay in subwavelength scale, and can find potential applications as filters, sensors, modulators and active optical delay lines in $\mathrm{THz}$ ultracompact circuits.
\end{abstract}

Keywords: plasmonic waveguide, plasmon-induced transparency, terahertz, slow light, graphene

\section{Introduction}

Terahertz (THz) is at the region between microwave and optical frequency, and related technologies have not been widely explored due to a lack of lab-based sources until mid-1980s [1]. In recent decades, significant progress has been made in efficient and compact $\mathrm{THz}$ sources, which leads to great demand for $\mathrm{THz}$ devices [2-3]. Especially, THz on-chip integrated circuits have drawn more attention owing to the potential applications in communication, imaging and chipscale sensing [4-6]. As a basic component of chipscale systems, $\mathrm{THz}$ waveguides have also been focused [7]. Up to now, various $\mathrm{THz}$ waveguides have been introduced such as polymer waveguides [8-9], silicon waveguides [10-14] and metallic waveguides [15-16]. However, the footprints of these conventional waveguides are restricted by the diffraction limit, therefore cannot meet the requirement of ultracompact circuits.

It is known that electromagnetic waves can propagate beyond the diffraction limit in the form of surface plasmon polaritons (SPPs) [17]. Generally, SPPs can exist on the dielectric-metal interface in optical and near-infrared frequencies, since waves within such frequencies can stimulate collective oscillations of electron plasma in the metal. However, metal will be a perfect electric conductor for $\mathrm{THz}$ waves and cannot support $\mathrm{THz}$ SPPs. To mimic SPPs, THz spoof SPPs are introduced based on corrugated metal surfaces [18-21]. However, spoof plasmonic waveguides suffer from integration difficulty, as well as nonarbitrary length due to the periodic structures [22]. Recently, it has been reported that semiconductors, such as Indium Antimonide (InSb), can support SPPs in THz band due to the similar optical property in THz with that of metals in optical band [23-25]. Resembling infrared plasmonic metal-insulator-metal (MIM) waveguides, $\mathrm{THz}$ plasmonic semiconductor-insulatorsemiconductor (SIS) waveguides are also introduced [26]. SIS waveguides can deliver $\mathrm{THz}$ waves in deep-subwavelength scale, meanwhile possess flexible integration and easy fabrication, consequently are suitable for highly integrated $\mathrm{THz}$ circuits.

Recently, plasmon-induced transparency (PIT), an analogue of the electromagnetically induced transparency (EIT) in three-level atomic system, has drawn more attention due to promising photonic applications [27-28]. PIT can produce the transparent peak via the destructive interference between directly-excited (bright) and indirectly-excited (dark) modes, and slow light effect can happen within the transparent window due to the intense dispersion [29]. Up to now, PIT-based slow light effect has been widely proposed based on metamaterials [30-32] and chipscale system [33-37], which can play an important role in optical signal buffering and time-domain processing [38].

However, there are few works to investigate $\mathrm{THz}$ PIT-based slow light effect in SIS waveguide system up to now. Therefore, in this work, we numerically and systematically investigate PIT as well as slow light effect based on $\mathrm{THz}$ plasmonic SIS waveguide system. Utilizing two side-coupled stub resonators, PIT is realized, and the induced strong dispersion within the transparent window will bring about the slow light. Via tuning the length or position of stubs, the profile of PIT can be flexibly modulated, and the slow light is tailored at the same time. Especially, PIT as well as slow light can also be actively adjusted by the temperature due to the temperature-dependent optical properties of InSb. Furthermore, by integrating monolayer graphene in the system, PIT and slow light can also be dynamically tuned via applying voltage on graphene. This work realizes geometrical, thermal and electrical approaches to manipulate $\mathrm{THz}$ transmission and group delay in subwavelength scale, and can find potential $\mathrm{THz}$ on-chip applications as filters, sensors, modulators and active optical delay lines. 
The proposed structure is shown in Fig. 1, including an input waveguide and two side-coupled stub microcavities. The geometric parameters are as follows: $w=50 \mu \mathrm{m}, L_{1}=280 \mu \mathrm{m}, L_{2}=$ $240 \mu \mathrm{m}, d=600 \mu \mathrm{m}$, and $D=d_{2}-d_{1}$, which is the distance between two stubs. In practice, such structure can be fabricated by applying laser micro-machining process on InSb wafer [39], and the measurement can be fulfilled via $\mathrm{THz}$ time-domain spectrometers [23]. The permittivity of InSb can be described as follows [23, 4042]:

$$
\varepsilon_{\text {InSb }}=\varepsilon_{\infty}-\frac{\omega_{p}^{2}}{\omega^{2}+i \gamma \omega}
$$

where $\varepsilon_{\infty}$ is the permittivity at infinite frequency, which is 15.68 . $\omega$ is the angular frequency of the incident wave, $\gamma$ represents damping constant, which is $0.1 \pi \mathrm{THz} . \omega_{p}$ is the plasma frequency and is defined as follows:

$$
\omega_{p}=\sqrt{N e^{2} / \varepsilon_{0} m^{*}}
$$

here, $e$ is the electronic charge, $\varepsilon_{0}$ is the permittivity of vacuum, and $m^{*}=0.015 m_{e}$, where $m^{*}$ is the effective mass of free carriers, and $m_{e}$ is the mass of electron, which is $9.1 \times 10^{-31} \mathrm{~kg}$. $N$ is the intrinsic carrier density, which can be calculated by the following equation:

$$
N=5.76 \times 10^{20} T^{1.5} \exp \left(-0.26 / 2 k_{B} T\right)
$$

where $k_{B}$ is the Boltzmann constant, and $T$ is the temperature in Kelvin (K). Obviously, the plasma frequency of $\mathrm{InSb}$ can be influenced by temperature. The real and imaginary part of permittivity for InSb under different temperatures from 0.6 to 1 THz are depicted in Fig. 2(a) and (b), respectively. It is shown that higher temperature will make the real part of permittivity decline, while will increase the imaginary part.

Similar with MIM waveguides, SIS waveguides only support THz SPPs with transverse-magnetic (TM) mode. Besides, there is only fundamental TM mode because the width of waveguide $w$ is much smaller than the incident wavelength [43]. The dispersion of the fundamental TM mode follows this equation [44]:

$$
\frac{\varepsilon_{i} \sqrt{\beta^{2}-\varepsilon_{\text {InSb }} k_{0}^{2}}}{\varepsilon_{\text {InSb }} \sqrt{\beta^{2}-\varepsilon_{i} k_{0}^{2}}}+\tanh \left(\frac{w \sqrt{\beta^{2}-\varepsilon_{i} k_{0}^{2}}}{2}\right)=0
$$

here, $\varepsilon_{i}$ is the permittivity of insulator, $k_{0}=2 \pi / \lambda_{0}$ is the wavevector in the free space. $\beta$ is the wavevector of SPPs in SIS waveguides, and the effective refractive index is $n_{\text {eff }}=\beta / k_{0}$. The

calculated real and imaginary part of $n_{\text {eff }}$ under different temperatures are shown in Fig. 2 (c) and (d), respectively. Apparently, SPPs in SIS waveguides possess a temperaturedependent optical property.

The monolayer graphene can be expressed by 2D surface conductivity model, the conductivity of which is $\sigma_{\text {gra }}=\sigma_{\text {inter }}+$ $\sigma_{\text {intra }}$, where $\sigma_{\text {inter }}$ and $\sigma_{\text {intra }}$ are interband and intraband conductivity, respectively. In $\mathrm{THz}$ region, the conductivity of graphene is described by Kubo formula [45]:

$$
\sigma_{\text {gra }} \approx \sigma_{\text {intra }}\left(\omega, E_{F}, \Gamma, T\right)=i \frac{e^{2} k_{B} T}{\pi \hbar^{2}(\omega+i \Gamma)}\left[\frac{E_{F}}{k_{B} T}+2 \ln \left(e^{-\frac{E_{F}}{k_{B} T}}+1\right)\right]
$$

here, $\hbar$ is the reduced Planck's constant, $E_{F}$ is the Fermi level. $\Gamma=$ $1 / \tau$ is the carrier scattering rate, where $\tau$ describes carrier relaxation time which is defined as $\tau=\mu E_{F} / e v_{F}^{2}$, and $\mu$ is the carrier mobility and $v_{F}$ is the Fermi velocity. Here, we assign $\mu=$ $3000 \mathrm{~cm}^{2} / V \cdot s$ and $v_{F}=1.1 \times 10^{6} \mathrm{~m} / \mathrm{s}$, which accord with the experimental measurements [46-47].
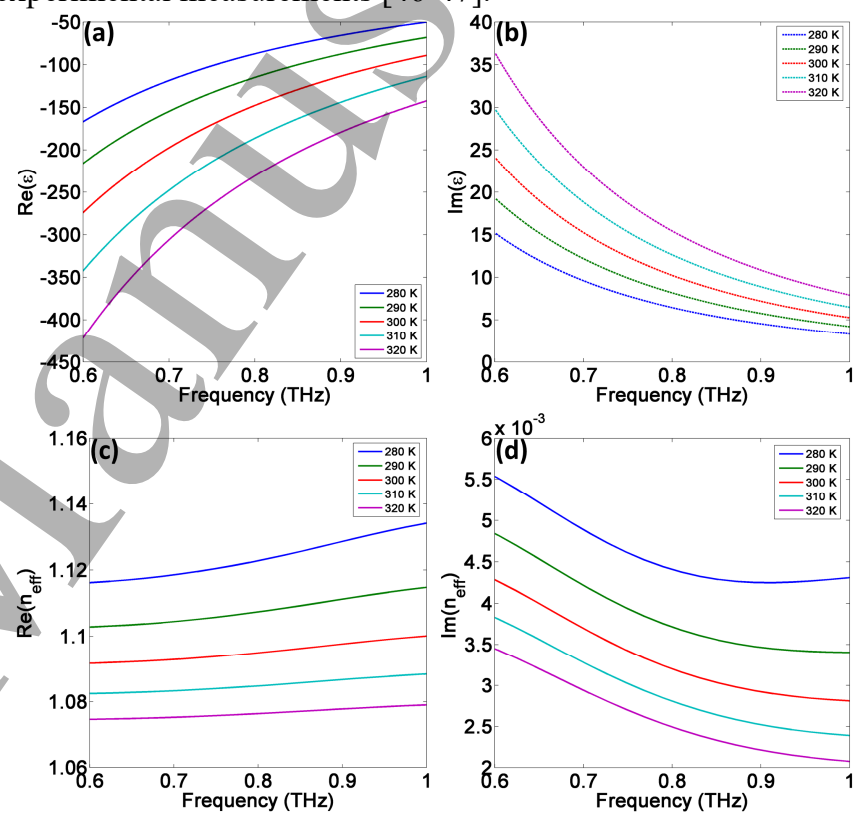

Fig. 2 (a) The real part of permittivity for InSb under different temperatures. (b) The imaginary part of permittivity for InSb under different temperatures. (c) The real part of effective refractive index under different temperatures. (d) The imaginary part of effective refractive index under different temperatures.

2D Lumerical Finite-Difference Time-Domain (FDTD) solution is utilized to do the simulation. In the domain of simulation, mesh sizes are both set as $1 \mu \mathrm{m}$ in $x$ - and $y$-direction to maintain the accuracy, and perfectly matched layers (PMLs) are used as absorbing boundary conditions to ensure the convergence. Plane wave source with y-direction electric field is set at $x=0$, and two power $(P)$ monitors are placed at $x=0$ and $x=d$ respectively. The transmission is calculated by $T=P_{\text {out }} / P_{\text {in }}$.

\section{Results and discussion}

The numerical transmission spectrum for three different structures are shown in Fig. 3(a). Here, we firstly consider $D=0$ $\mu \mathrm{m}$ and the temperature $T$ is kept as $300 \mathrm{~K}$. When only single upper stub (stub I) exists, there is one transmission dip at $0.71 \mathrm{THz}$ as shown in blue line in Fig. 3(c), and the corresponding $|\boldsymbol{H}|$ field distribution is given in Fig. 3(e). There will also be one dip at 0.81 $\mathrm{THz}$ if only single lower stub (stub II) exists as depicted in green 
line, and the corresponding field distribution is shown in Fig. 3(g). Such stub microcavity can be regarded as a semi Fabry-Perot (F$\mathrm{P})$ resonator, and the resonant condition is as follows [48]:

$$
\left(2 n+1-\frac{\varphi}{\pi}\right) \lambda=4 \operatorname{Re}\left(n_{e f f}\right) L, n=0,1 \ldots
$$

where $n$ is the mode order, which is an integer. $\varphi$ is the phase shift caused by the reflection on the interface between insulator and InSb at the end of the stub. $\lambda$ is the resonant wavelength, and $L$ is the length of the stub. The field distributions indicate that for both cases, $n=1$.
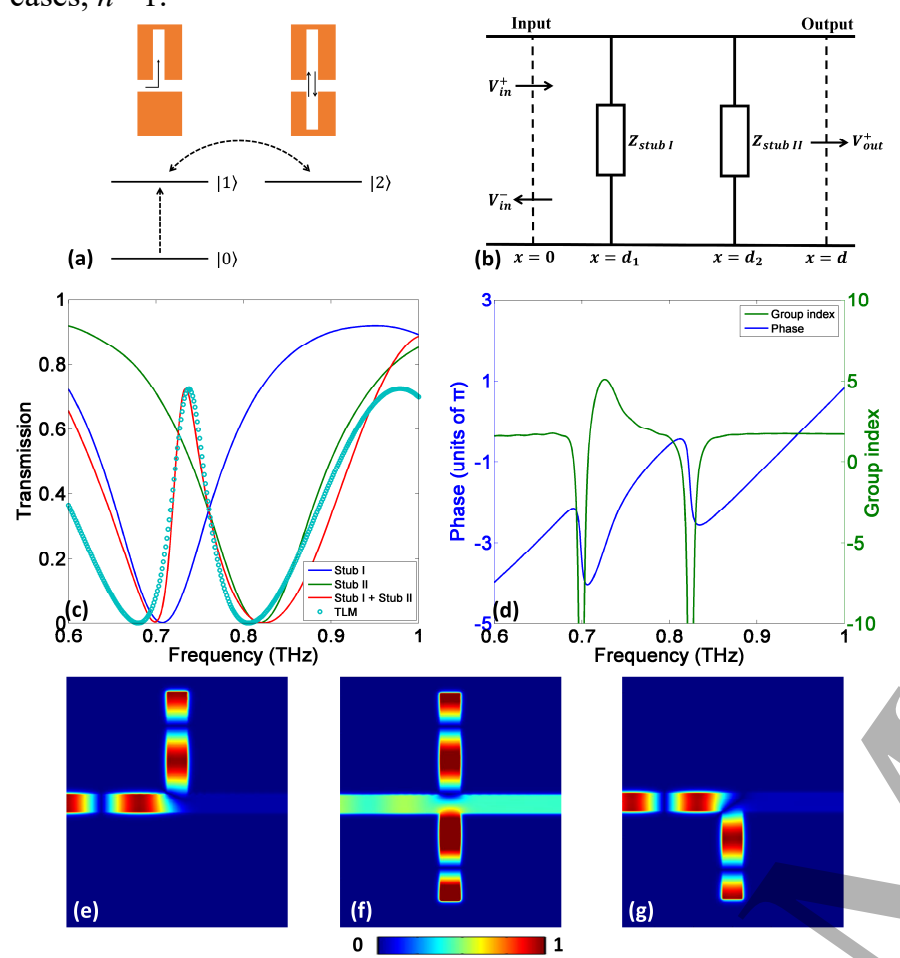

Fig. 3 (a) The analogue of three-level atom EIT system. (b) The equivalent transmission-line model. (c) The numerical transmission spectrum for three different cases: only stub I, only stub II as well as a combination of stub I and II, and the PIT transmission spectra from TLM. (d) The transmission phase shift and group delay line of the double stub system. (e) The $|\mathbf{H}|$ field distribution at 0.71 $\mathrm{THz}$ when there is only stub I. (f) The $|\mathbf{H}|$ field distribution at $0.73 \mathrm{THz}$ when there are double stubs. (g) The $|\mathbf{H}|$ field distribution at $0.81 \mathrm{THz}$ when there is only stub II.

When stub I and II are introduced simultaneously as shown in Fig. 1 when $D=0 \mu \mathrm{m}$, PIT happens as presented in red line in Fig. 3(c). There is a transparent peak at $0.73 \mathrm{THz}$, which is PIT effect. Here, a single stub microcavity can be directly excited and serves as the bright mode, while F-P resonance can be formed between the two stubs, behaving as the dark mode in the PIT system [49]. Therefore, such system constructs an analogue of three-level atom system as shown in Fig. 3(a), and the transparent peak is induced via the destructive interference between bright and dark modes. The corresponding field distribution is shown in Fig. 3(f).

Considering that the width of SIS waveguide is much smaller than the THz wavelength and only single fundamental mode exist, such transmission spectrum of PIT can also be theoretically described by the transmission-line method (TLM), which is the photonic analogy of subwavelength waveguiding in electronics [50], and the equivalent transmission-line model is shown in Fig. 3(b). In this model, the electric and magnetic field of SPPs are analogized by the voltage $V$ and current $I$, and the waveguide as

well as stub resonators are characterized by the effective impedances $Z$. For the waveguide, which is the infinite transmission line, the corresponding effective impedance is as follows [51]:

$$
Z_{S I S} \approx \frac{E_{y} w}{H_{z}}=\frac{\beta w}{\omega \varepsilon_{0} \varepsilon_{i}}
$$

here, $\varepsilon_{0}$ is the permittivity of vacuum, $E_{y}$ and $H_{z}$ are y-component electric field and z-component magnetic field of SPPs, respectively. For the stub resonator, which is a finite transmission line, the effective impedance is given by a $Z_{S I S}$ terminated by a $\operatorname{load} Z_{L}$ :

$$
Z_{\text {stub } \mathrm{n}}=Z_{S I S} \frac{Z_{L}-i Z_{S I S} \tan \left(\beta L_{n}\right)}{Z_{S I S}-i Z_{L} \tan \left(\beta L_{n}\right)}, \quad n=I, I I
$$

where $Z_{L}=\sqrt{\varepsilon_{I n S b} / \varepsilon_{i}} Z_{S I S}$, accounting for damping and phase shift of SPPs caused by the reflection at the end of the stub. According to transfer matrix method [52], the input (at $x=0$ ) and output (at $x=d$ ) voltages are related as follows:

$$
\left(\frac{V_{\text {in }}^{+}}{V_{\text {in }}^{-}}\right)=\boldsymbol{T}\left(\frac{V_{\text {out }}^{+}}{0}\right)
$$

where the transfer matrix $\boldsymbol{T}$ is described by:

$$
\boldsymbol{T}=\boldsymbol{A}\left(d_{1}\right) \boldsymbol{B}\left(Z_{\text {stub I }}\right) \boldsymbol{A}(D) \boldsymbol{B}\left(Z_{\text {stub II }}\right) \boldsymbol{A}\left(d-d_{2}\right)
$$

here, the transfer matrix $\boldsymbol{A}(\mathrm{x})$ refers to the SPP propagation in the waveguides, while the matrix $\boldsymbol{B}$ (stub) describes the coupling between backward- and forward-propagating SPPs induced by the effective impedance $Z_{\text {stub }}$. The detail expressions of A and B can be found in [51]. Finally, the transmission can be calculated by:

$$
T=\left|\frac{V_{\text {out }}^{+}}{V_{\text {in }}^{+}}\right|^{2}=\left|\left(1+\frac{Z_{\text {SIS }}}{2 Z_{\text {stub I }}}\right)\left(1+\frac{Z_{\text {SIS }}}{2 Z_{\text {stub II }}}\right)-\frac{Z_{S I S}^{2} \exp (2 i \beta D)}{4 Z_{\text {stub I }} Z_{\text {stub II }}}\right|^{-2} \exp \left(-\frac{d}{d_{\text {spp }}}\right)
$$

where $d_{s p p}=\lambda_{0} /\left(4 \pi \operatorname{Im}\left(n_{\text {eff }}\right)\right)$. The transmission spectra calculated from TLM with $D=0 \mu \mathrm{m}$ is plotted in Fig. 3(a), showing a good fitting with numerical results.

Meanwhile, such sharp peak indicates a strong dispersion within the transparent window, which will manipulate the transmission phase and lead to the slow light effect. The group delay of slow light can be assessed by the group index $n_{g}[53]$ :

$$
n_{g}=\frac{c}{d} \times \frac{d \psi(\omega)}{d \omega}
$$

where $c$ is the light speed, and $d$ is the length of the device. $\psi(\omega)$ is the transmission phase shift and is provided in blue line in Fig. 3(d). The group index is illustrated in green line in Fig. 3(d), and there is a relatively higher group index 5.11 at the position of transparent peak, which indicates the emergence of group delay, i.e., the slow light effect.

The impacts brought by geometric parameters on PIT profile as well as group delay line are investigated. At first, the length of stub I $L_{1}$ is changed from 260 to $300 \mu \mathrm{m}$, while the length of stub II $L_{2}$ remains at $240 \mu \mathrm{m}$. The corresponding transmission spectrum in Fig. 4(a) show that, with the increasing $L_{1}$, the transparent peak will have a red shift, the full width at half maximum (FWHM) of the peak will be wider, and the peak amplitude will rise. Such profile evolution comes from the change of resonant frequency detuning between the two coupled resonators. Meanwhile, as illustrated in Fig. 4(b), the position of group delay will follow the 
peak. However, wider peak will lead to the decline of the group index within it, which is up to 9.45 when $L_{1}=260 \mu \mathrm{m}$ while only 3.40 when $L_{l}=300 \mu \mathrm{m}$. This is because narrower peak will be sharper, consequently brings about a stronger dispersion within the transparent window [54]. Fig. 4(c) and (d) present the transmission spectrum as well as group index line when changing $L_{2}$ from 220 to $260 \mu \mathrm{m}$, while $L_{1}$ is kept as $280 \mu \mathrm{m}$. It is shown that longer $L_{2}$ will also lead to a red shift of peak, however, the peak will be narrower and lower. The induced behavior of group index is similar as depicted in Fig. 4(d), and the group index at the peak can reach 10 when $L_{2}=260 \mu \mathrm{m}$. Therefore, the profile of PIT as well as group delay line can be tailored via changing the length of stubs. However, there is a trade-off between the transmittance and group index at the peak.
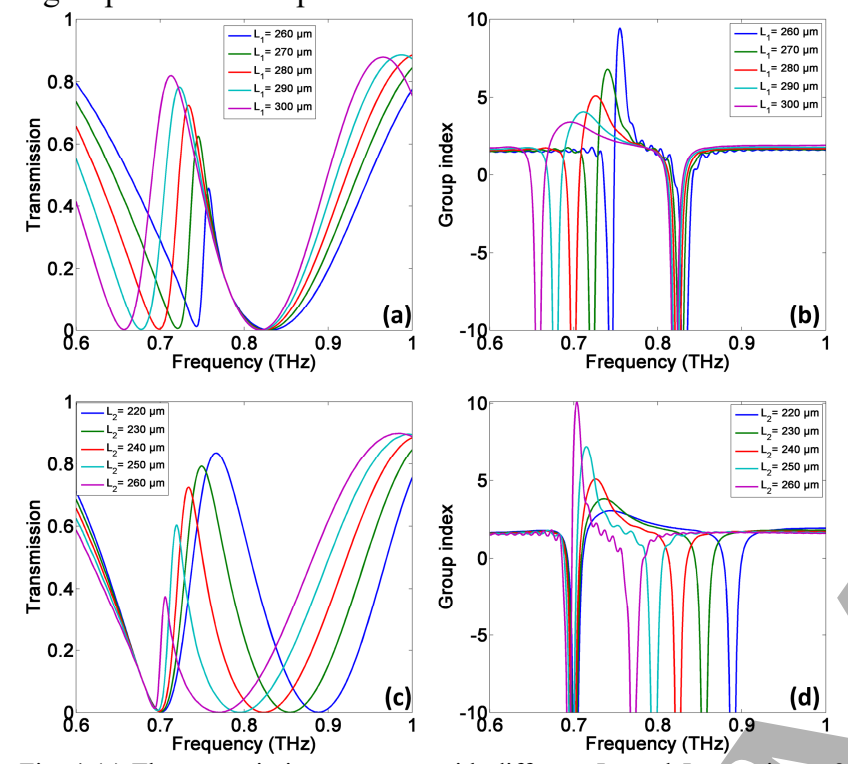

Fig. 4 (a) The transmission spectrum with different $L_{1}$, and $L_{2}$ remains at 240 $\mu \mathrm{m}$. (b) The group delay line with different $L_{1}$, and $L_{2}$ remains at $240 \mu \mathrm{m}$. (c) The transmission spectrum with different $L_{2}$, and $L_{1}$ remains at $280 \mu \mathrm{m}$. (d) The group delay line with different $L_{2}$, and $L_{1}$ remains at $280 \mu \mathrm{m}$.

Besides, the influences of the distance $D$ between the two stubs in the $x$ direction are also studied. Here, $L_{1}$ and $L_{2}$ remain at 280 and $240 \mu \mathrm{m}$ respectively, $T$ is kept as $300 \mathrm{~K}$, and $D$ is varied from 0 to $50 \mu \mathrm{m}$. Fig. 5(a) shows that the amplitude of transparent peak will drop with increasing $D$, while the position of peak will remain unchanged. This is because SPPs will accumulate phase when propagating between the two stubs, which is described by $\phi=\omega n_{\text {eff }} D / c$. The phase match between the two coupled resonators will be modified, which in turn affects the interference process [55]. Notably, the second term of Eq. (11) also describes such interference process [51]. In the meantime, the group index at the peak position will also decline from 5.11 to 2.68 without any frequency shift. Therefore, the amplitude of PIT as well as slow light can be tailored via changing the distance between the two stubs.

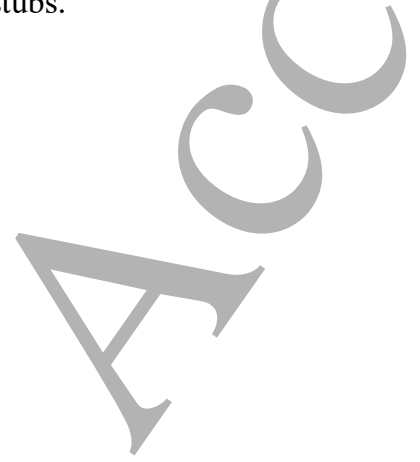

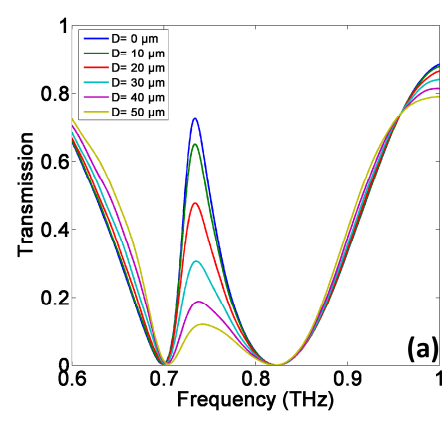

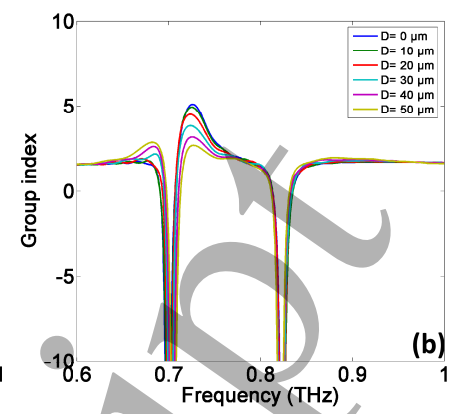

Fig. 5 (a) The transmission spectrum with different $D$. (b) The group delay line with different $D$.

Furthermore, we also investigate the relation between temperatures and PIT as well as slow light. Fig. 6(a) shows the transmission spectrum under different temperatures, $L_{1}$ and $L_{2}$ are kept at 280 and $240 \mu \mathrm{m}$, respectively, and $D=0 \mu \mathrm{m}$. It is shown that the whole spectrum will have a blue shift with increasing temperature, and the profile of PIT is almost unchanged except for a little rise of the transparent peak. The shift is due to the decrease of $\operatorname{Re}\left(n_{\text {eff }}\right)$ of the fundamental mode, and the increasing peak attributes the decrease of $\operatorname{Im}\left(n_{\text {eff }}\right)$, which indicates lower loss. Simultaneously, the group index will also have a blue shift as given in Fig. 6(b). Since there is little change of PIT profile, the group index at the transparent peak is stable. Therefore, active control of the position of PIT as well as slow light can be realized via changing the ambient temperature.
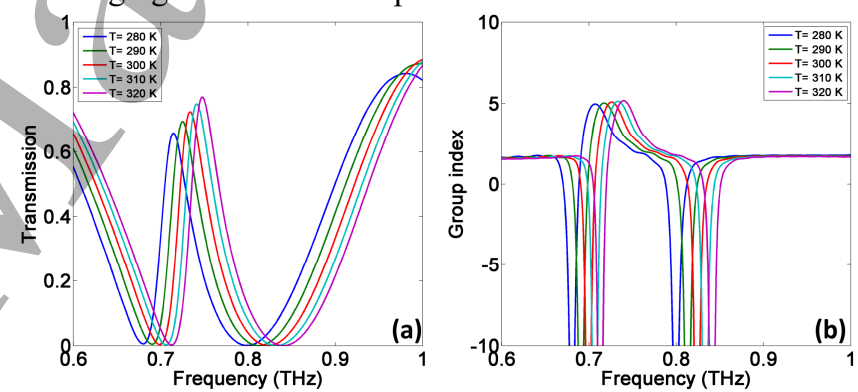

Fig. 6 (a) The transmission spectrum with different ambient temperatures. (b) The group delay line with different ambient temperatures.

Finally, a monolayer graphene is integrated into this system as shown in the inset of Fig. 7(a). Via applied voltage, the Fermi level of graphene can be actively tuned [56]. Here, $L_{1}$ and $L_{2}$ are still 280 and $240 \mu \mathrm{m}$ respectively, $D=0 \mu \mathrm{m}$, and $T$ is kept at $300 \mathrm{~K}$. The transmission spectrum under different Fermi levels of graphene are shown in Fig. 7(a), and the corresponding different group index are depicted in Fig. 7(b). It is shown that the increasing Fermi level, from $0.2 \mathrm{eV}$ to $0.6 \mathrm{eV}$, will significantly suppress the transmission peak from $73 \%$ to $15 \%$. The $|\mathbf{H}|$ field distributions at the transparent peak when there is no graphene, graphene with $0.3 \mathrm{eV}$ and $0.6 \mathrm{eV}$ are presented in Fig. 7(c-e) respectively, from which we can see that the energy is partly reflected as well as consumed on the interface of graphene, which attributes to the fact that graphene becomes more conductive with the rise of Fermi level, meanwhile more loss is also introduced on the graphene surface. Meanwhile, the group index within the peak can be lifted from 5.11 to 7.35 due to the modification of the dispersion within the transparent window, however, suffering a low transmission. Here, we just provide a proof of the concept for the active modulation of graphene in this structure. In practice, the 
SIS waveguide can be filled with low loss materials in $\mathrm{THz}$ such as silicon [57], and graphene can be embedded into it. Such graphene-loaded structure can be utilized as an active filter, modulator and spacer in $\mathrm{THz}$ chipscale system.
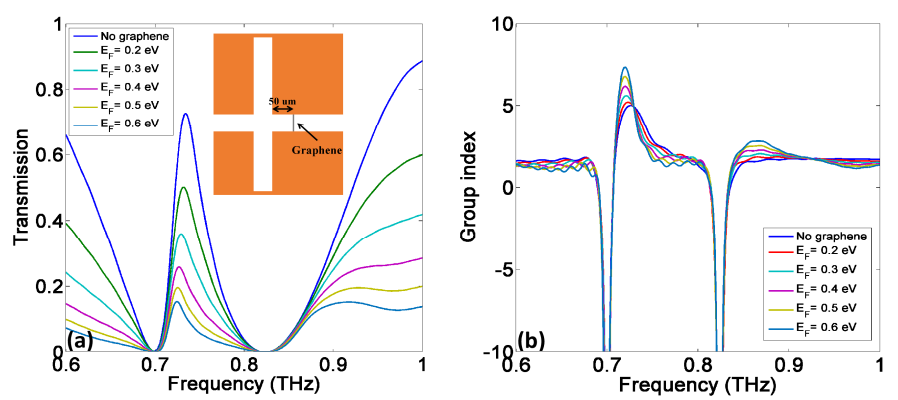
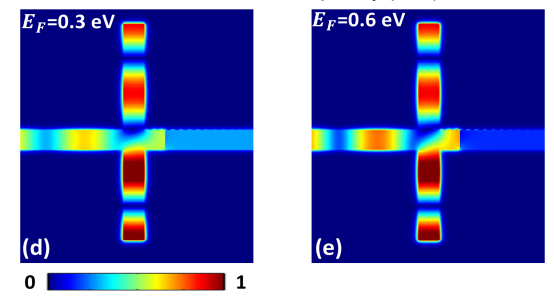

Fig. 7(a) The transmission spectrum under different Fermi levels of graphene. The graphene-embedded system is shown in the inset. (b) The group delay line under different Fermi levels of graphene. (c) The $|\mathbf{H}|$ field distribution at $0.71 \mathrm{THz}$ when there is no graphene. (d) The $|\mathbf{H}|$ field distribution at $0.71 \mathrm{THz}$ when Fermi level of graphene is $0.3 \mathrm{eV}$. (e) The $|\mathbf{H}|$ field distribution at $0.71 \mathrm{THz}$ when Fermi level of graphene is $0.6 \mathrm{eV}$.

\section{Conclusion}

In summary, we numerically propose the slow light tailor based on the $\mathrm{THz}$ plasmonic SIS waveguide system. Utilizing two simple stub resonators, PIT is introduced to realize the group delay within the transparent window. Via tuning the geometric parameters, the PIT profile as well as group delay line can be adjusted at will. Besides, the position of PIT as well as slow light can be actively controlled by the ambient temperature. Furthermore, by embedding monolayer graphene into the waveguide, the applied voltage can be utilized to modulate the amplitude of the transparent peak. This work introduces geometrical, thermal and electrical control of $\mathrm{THz}$ transmission and group delay in subwavelength scale and have potential applications in $\mathrm{THz}$ highly integrated circuits such as filters, sensors, modulators and active optical delay lines.

\section{Acknowledgments}

This work is supported by the Foundation of NUDT (ZK17-0301 ) and the Program for New Century Excellent Talents in University (NCET-12-0142).

\section{Conflicts of Interest}

The authors declare no conflict of interest.

\section{References}

[1] Tonouchi, Masayoshi. "Cutting-edge terahertz technology." Nature photonics., vol. 1, no. 2, pp. 97, 2007.

[2] Siegel, Peter H. "Terahertz technology." IEEE Transactions on microwave theory and techniques., vol. 50, no. 3, pp. 910-928, 2002.

[3] Sakai, Kiyomi, ed. Terahertz optoelectronics. Vol. 6. Berlin: Springer, 2005.

[4] Nagatsuma, Tadao, Guillaume Ducournau, and Cyril C. Renaud. "Advances in terahertz communications accelerated by photonics." Nature Photonics., vol. 10, no. 6, pp. 371, 2016.

[5] Guerboukha, Hichem, Kathirvel Nallappan, and Maksim Skorobogatiy. "Toward real-time terahertz imaging." Advances in Optics and Photonics., vol. 10 , no. 4, pp. 843-938, 2018

[6] Nagel, M., M. Först, and H. Kurz. "THz biosensing devices: fundamentals and technology." Journal of Physics: Condensed Matter., vol. 18, no. 18, pp. S601, 2006

[7] Atakaramians, Shaghik, et al. "Terahertz dielectric waveguides." Advances in Optics and Photonics., vol. 5, no. 2, pp. 169-215, 2013.

[8] Goto, Masahiro, et al. "Teflon photonic crystal fiber as terahertz waveguide." Japanese Journal of Applied Physics., vol. 43, no. 2B, pp. L317, 2004.

[9] Hidaka, Takehiko, et al. "Ferroelectric PVDF cladding terahertz waveguide." Journal of Lightwave Technology., vol. 23, no. 8, pp. 24692473, 2005.

[10] Vogt, Dominik Walter, and Rainer Leonhardt. "Ultra-high Q terahertz whispering-gallery modes in a silicon resonator." APL Photonics., vol. 3, no. 5, pp. 051702, 2018.

[11] Xie, Jingya, et al. "Terahertz integrated device: high-Q silicon dielectric resonators." Optical Materials Express., vol. 8, no. 1, pp. 50-58, 2018.

[12] Gao, Weijie, et al. "Effective-medium-cladded dielectric waveguides for terahertz waves." Optics Express 27.26 (2019): 38721-38734.

[13] Headland, Daniel, et al. "Near-field out-of-plane coupling between terahertz photonic crystal waveguides." Optica 6.8 (2019): 1002-1011.

[14] Sørgård, Trygve, et al. "Terahertz waveguiding in glass-clad silicon wafers." Optical Materials Express 10.3 (2020): 742-751.

[15] Wang, Kanglin, and Daniel M. Mittleman. "Metal wires for terahertz wave guiding." Nature., vol. 432, no. 7015, pp. 376, 2004.

[16] Jeon, Tae-In, Jiangquan Zhang, and D. Grischkowsky. "THz Sommerfeld wave propagation on a single metal wire." Applied Physics Letters., vol. 86, no. 6, pp. $161904,2005$.

[17] Gramotnev, Dmitri K., and Sergey I. Bozhevolnyi. "Plasmonics beyond the diffraction limit." Nature photonics., vol. 4, no. 2, pp. 83, 2010.

[18] Gao, Zhen, et al. "Spoof plasmonics: from metamaterial concept to topological description." Advanced Materials., vol. 30, no. 31, 1706683, 2018.

[19] Zhang, Xueqian, et al. "Terahertz surface plasmonic waves: a review." Advanced Photonics 2.1 (2020): 014001.

[20] Yuan, Mingrui, et al. "High-performance and compact broadband terahertz plasmonic waveguide intersection." Nanophotonics 8.10 (2019): 1811-1819.

[21] Yuan, Mingrui, et al. "Curved terahertz surface plasmonic waveguide devices." Optics Express 28.2 (2020): 1987-1998.

[22] Jingiing Zhang, et al. "Integrated spoof plasmonic circuits." Journal of Science Bulletin., vol. 64, no. 12, pp. 843-855, 2019.

[23] Rivas, Jaime Gómez, et al. "Transmission of THz radiation through InSb gratings of subwavelength apertures." Optics express., vol. 13, no. 3, pp. 847-859, 2005.

[24] Hanham, S. M., et al. "Broadband terahertz plasmonic response of touching InSb disks." Advanced Materials, vol. 24, no. 35, pp. OP226-OP230, 2012

[25] Giannini, Vincenzo, et al. "Scattering efficiency and near field enhancement of active semiconductor plasmonic antennas at terahertz frequencies." Optics express., vol. 18, no. 3, pp. 2797-2807, 2010.

[26] Tao, Jin, et al. "Tunable subwavelength terahertz plasmonic stub waveguide filters." IEEE Transactions on Nanotechnology., vol. 12, no. 6, pp. 1191 $1197,2013$.

[27] Niu, Xinxiang, et al. " Plasmon-induced transparency effect for ultracompact on-chip devices." Nanophotonics 8.7 (2019): 1125-1149.

[28] Sarkar, Rakesh, et al. "Polarization independent double-band electromagnetically induced transparency effect in terahertz metamaterials." Journal of Optics 22.3 (2020): 035105.

[29] Zhang, Shuang, et al. "Plasmon-induced transparency in metamaterials." Physical review letters 101.4 (2008): 047401.

[30] Sarkar, Rakesh, et al. "independently tunable electromagnetically induced transparency effect and dispersion in a multi-band terahertz metamaterial." Scientific Reports 9.1 (2019): 1-10.

[31] Wang, Qiao, et al. "Electromagnetically induced transparency in an alldielectric nano-metamaterial for slow light application." Optics Express 27.24 (2019): 35012-35026.

[32] Zhao, Zhenyu, et al. "Demonstration of group delay above $40 \mathrm{ps}$ at terahertz plasmon-induced transparency windows." Optics express 27.19 (2019): 26459-26470.

[33] Tang, Baojie, et al. "Plasmonic-induced transparency and unidirectional control based on the waveguide structure with quadrant ring resonators." Applied Physics Express 8.3 (2015): 032202.

[34] Song, Ci, et al. "Wavelength-sensitive PIT-like double-layer graphenebased metal-dielectric-metal waveguide." Applied optics 57.33 (2018) 9770-9776. 
[35] Liu, Dongdong, et al. "Plasmon-induced transparency and refractive index sensing based on a trapezoid cavity coupled with a hexagonal resonator." Plasmonics 14.3 (2019): 663-671

[36] Tian, Jinping, and Jiejin Li. "Investigation on Plasmon Induced Transparency and its Application in an MIM Typecompound Plasmonic Waveguide." Progress In Electromagnetics Research 98 (2020): 199-212.

[37] Li, Hongju, et al. "Strong plasmon-exciton coupling in MIM waveguideresonator systems with WS 2 monolayer." Optics Express 28.1 (2020): 205 215 .

[38] Krauss, Thomas F. "Why do we need slow light?." Nature Photonics 2.8 (2008): 448 .

[39] Bhatt, Piyush, et al. "Bio-interfacing of resonant transmission characteristics of InSb-based terahertz plasmonic waveguide." Biomedical Physics \& Engineering Express 1.2 (2015): 025003.

[40] Howells, S. C., and L. A. Schlie. "Transient terahertz reflection spectroscopy of undoped InSb from 0.1 to 1.1 THz." Applied Physics Letters 69.4 (1996): 550-552.

[41] Sánchez-Gil, José A., and Jaime Gomez Rivas. "Thermal switching of the scattering coefficients of terahertz surface plasmon polaritons impinging on a finite array of subwavelength grooves on semiconductor surfaces." Physical Review B., vol. 73, no. 20, pp. 205410, 2006.

[42] Han, Jiaguang, and Akhlesh Lakhtakia. "Semiconductor split-ring resonators for thermally tunable terahertz metamaterials." Journal of Modern Optics., vol. 56, no. 4, pp. 554-557, 2009.

[43] Dionne, J. A., et al. "Plasmon slot waveguides: Towards chip-scale propagation with subwavelength-scale localization." Physical Review B., vol. 73 , no. 3 , pp. $035407,2006$.

[44] Gordon, Reuven, and Alexandre G. Brolo. "Increased cut-off wavelength for a subwavelength hole in a real metal." Optics Express., vol. 13, no. 6, pp. 1933-1938, 2005.

[45] Hanson, G. W. "Dyadic Green's functions and guided surface waves for a surface conductivity model of graphene. " Journal of Applied Physics, 103(6), 064302, 2008.

[46] Zhang, Y., Tan, Y. W., Stormer, H. L., \& Kim, P. "Experimental observation of the quantum Hall effect and Berry's phase in graphene." nature, 438(7065), 201, 2005.

[47] Jnawali, G., Rao, Y., Yan, H., \& Heinz, T. F. "Observation of a transient decrease in terahertz conductivity of single-layer graphene induced by ultrafast optical excitation. " Nano letters, 13(2), 524-530, 2013.

[48] Lin, Xian-Shi, and Xu-Guang Huang. "Tooth-shaped plasmonic waveguide filters with nanometeric sizes." Optics letters., vol.33, no. 23, pp. 2874-2876, 2008.

[49] Wang, B., Zeng, Q., Xiao, S., Xu, C., Xiong, L., Lv, H., ... \& Yu, H. "Lowpower, ultrafast, and dynamic all-optical tunable plasmon induced transparency in two stub resonators side-coupled with a plasmonic waveguide system.” Journal of Physics D: Applied Physics, 50(45), 455107 2017.

[50] Kocabas, Sukru Elin, et al. "Transmission line and equivalent circuit models for plasmonic waveguide components." IEEE Journal of Selected Topics in Quantum Electronics 14.6 (2008): 1462-1472.

[51] Pannipitiya, Asanka, et al. "Improved transmission model for metaldielectric-metal plasmonic waveguides with stub structure." Optics express 18.6 (2010): 6191-6204.

[52] Liu, Jianlong, et al. "Surface plasmon reflector based on serial stub structure." Optics express 17.22 (2009): 20134-20139.

[53] Lu, Hua, Xueming Liu, and Dong Mao. "Plasmonic analog of electromagnetically induced transparency in multi-nanoresonator-coupled waveguide systems." Physical Review A., vol. 85, no. 5, pp. 053803, 2012.

[54] He, Jinna, et al. "Gain-assisted plasmon induced transparency in T-shaped metamaterials for slow light." Journal of Optics., vol. 17, no. 5, pp. 055002 , 2015.

[55] Kekatpure, Rohan D., et al. "Phase-coupled plasmon-induced transparency." Physical review letters., vol. 104, no. 24, pp. 243902, 2010.

[56] Ju, L., Geng, B., Horng, J., Girit, C., Martin, M., Hao, Z., ... \& Wang, F. "Graphene plasmonics for tunable terahertz metamaterials." Nature nanotechnology, 6(10), 630, 2011.

[57] Withayachumnankul, W., Fujita, M., \& Nagatsuma, T. "Integrated silicon photonic crystals toward terahertz communications. " Advanced Optical Materials, 6(16), 1800401, 2018. 